\title{
Research Advances of Aeolian Vibration of Damper-Line System
}

\author{
Wenping Xie, Tian Peng, Kai Xiao, and Jian Zhang
}

\begin{abstract}
Aeolian vibration limits the improvement of the stress of the conduct wire, which makes the conductor high cost. the current situation that transmission line cannot just rely on it self- damping to dissipate the wind energy input, anti-vibration measures such as vibration fittings are applied in our country. But transmission line and damper system cannot completely protect the lines from the destruction of Aeolian vibration. For the sake of further studies of Aeolian vibration, the development process of the research on the Aeolian vibration of damper-line system is described based on the summary of relevant research progresses on home and abroad in the recent decades. The causes of formation and mechanism of the Aeolian vibration are introduced in this paper. Some problems in the research are discussed in view of experimental study, fatigue life analysis, on-line monitoring. Then the development trend of the research about these aspects is prospected, which given the thoughts to other researchers.
\end{abstract}

Index Terms-Transmission line, Aeolian vibration, laboratory experiments, vibration fatigue, on-line monitoring.

\section{INTRODUCTION}

With the rapid development of China's economy and society and the rapid development of electricity demand, there is increasing pressure for the transmission network. The requirements of transmission lines tend to be higher and softer which make the diameter of wire diameter and span larger, transmission line suspension points higher. But Aeolian vibration limits the improvement of the stress of the conduct wire, which makes the conductor high cost. Due to the current situation that transmission line cannot just rely on it selfdamping to dissipate the wind energy input, anti-vibration measures such as vibration fittings are applied in our country , and strock-bridge damper, damper line, spacer damper are the main anti-vibration fittings.

According to the domestic and abroad operation experience of transmission line in recent decades, transmission line and damper system cannot completely protect the lines from the destruction of Aeolian vibration, although it have obvious improvement effect to suppress

Manuscript received September 8, 2016; revised November 12, 2016 This work was supported in part by Electric Power Research Institute of Guangdong Power Grid Co., Ltd, Guangzhou, 510080, China, and Tongji University, Shanghai, 200092, China.

W. Xie is with Guangdong Electric Power Company Research Institute, Guangzhou, 510030, China (e-mail: 309577110@qq.com).

T. Peng and J. Zhang is with the Department of Civil Engineering, Tongji University, Shanghai, 200092, China (e-mail: pt0796@163.com, 993139097@qq.com).

K. Xiao was with the Department of Materials Science and Engineering, Zhejiang University, Hangzhou, 310000, China. He is with Guangdong Electric Power Company Research Institute, Guangzhou, 510030, China (e-mail: 13928750816@163.com). vibration. Eventually, this leads to fatigue damage of the conductor in the form of broken strands in the outer layer, usually at the point of contact such as the suspension clamp. Also, there are other damage types including the slippage of the messenger wire of the Stockbridge damper, insulator strings, and the loosening of parts, as shown in Fig. 1.

This article are introduced about the development process of the research on the Aeolian vibration of damper-line system based on the summary of relevant research progresses on home and abroad in the recent decades, and the causes of formation and mechanism of the Aeolian vibration. There is more, the development trend of the research is current research of experimental study, fatigue life analysis, on-line monitoring are introduced, what is more, the development trend of the research is prospected according to demand of social and economic development.

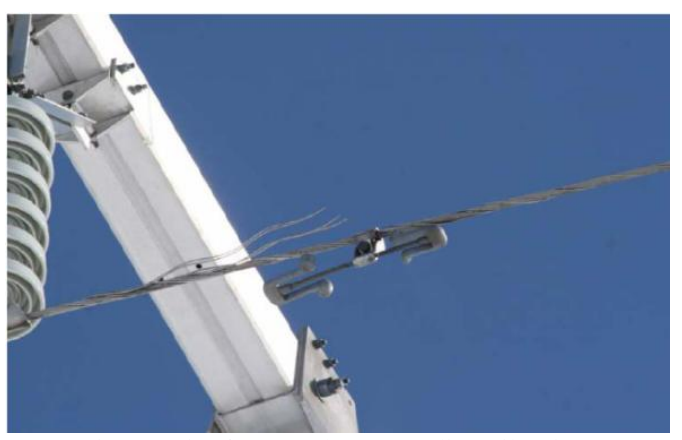

Fig. 1. Wire fracture due to Aeolian vibration.

\section{CAUSES OF Formation AND MECHANISM OF THE AEOLIAN VIBRATION}

Aeolian vibration is caused by alternating vortices. When the steady air flow to cylinder, turbulence will be produce in the back of the cylinder. Turbulence appeared up and down alternately and rotated in opposite directions (Fig. 2). When A point occurred vortex, the cylinder will appear the downward impulsive force for the reason of negative pressure and the speed of point B larger than point A; on the contrary, it will generate the upward impulsive force.

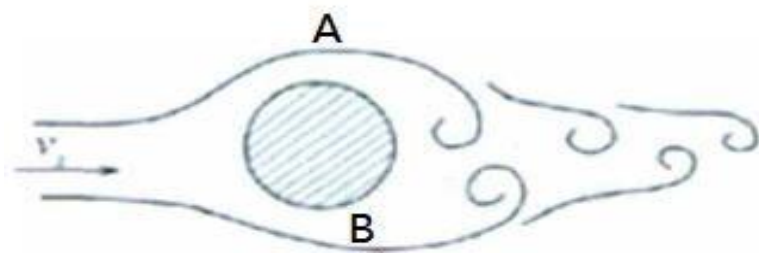

Fig. 2. Von Karman vortices.

Aeolian vibration known as wind-induced vibration, has a high frequency and small-amplitude motion, which arises 
from alternating forces caused by vortex shedding. The frequency of Aeolian vibration ranges from 3 to $50 \mathrm{~Hz}$ and the wind speed ranges from 1 to $7 \mathrm{~m} / \mathrm{s}[1]$-[3].The amplitude of Aeolian vibration less than diameter of the conductor, which ranges from 6 to $50 \mathrm{~mm}$. This force is periodic with a frequency $f_{s}$, which is related to the diameter of the conductor and the wind velocity as follows:

$$
f_{s}=S \frac{v}{d}
$$

where $s$ is the Strouhal number and it varies from 0.15 to 0.25 . In general the average Strouhal value is taken as 0.2 .

The theoretical natural frequency of a conductor without self-damper and a damper can be calculated as follows:

$$
f_{n}=\frac{n}{2 l} \sqrt{\frac{T_{0}}{m}}
$$

Equation (3) is the theoretical natural frequency of a conductor without a damper.

$$
f_{n}=\frac{n}{2 l} \sqrt{\frac{T_{0}}{m}} \sqrt{1+\frac{E J}{T_{0}\left(\frac{n \pi}{l}\right)^{2}}}
$$

where $n$ is the mode of natural frequency, $l$ is the length of conductor, $T_{0}$ is the tension of the conductor, $m$ is mass per unit length, $E J$ is the stiffness of the conductor. It is found that the error of the actual result of equation (2) and (3) will not more than $5 \%$, so the theoretical natural frequency of a conductor can be calculated ignored the stiffness of the conductor. The actual natural frequency of transmission line corresponds to different orders, so there are different frequencies, that is, the natural frequency is not a value, but a set of values.

It is noted that the conductor becomes very excited as the Strouhal frequency $f_{s}$ approaches some of its natural frequencies $f_{n}$ and this phenomena is called resonance, then the Aeolian vibration happen. There is a self-locking phenomenon according the actual observation of the Aeolian vibration of transmission line. That is, the vortex frequency and the natural frequency of conductor will not change and still keep the natural frequency of the overhead line although the wind speed change in a certain range.

\section{EXPERIMENTAL STUDY OF AEOLIAN VIBRATION AND DYNAMIC RESEARCH}

\section{A. Wind Tunnel Experimental Study}

The energy balance method is the most popular concept used to predict the vibration of transmission lines because it is simple, easy to understand, the relationship is clear and the vibration amplitude can be calculated. Scholars at home and abroad carried out a large number of wind tunnel experiments to measure value of input wind power of transmission line. Rawlins C. [4], Macdonald R. [5], Brika D. [6] and Brika D. [7] given the experimental curves to characterize the variation of wind power which can be expressed as the complex function about amplitude, frequency, wind speed, the diameter of the transmission line and more parameters which need to be determined by experiment. Kraus M. [8] measured transmission lines operating wind power, then compared with the wind tunnel experimental results and made the conclusions: confirmed the current wind power input curve and the wind power input affected by wind turbulence intensity seriously. Deyi K. [9] refer the classic wind tunnel experiment, researched the wind power input function of transmission line under the different turbulent intensity by means of numerical wind tunnel technology to provide the parameterization exciting force for the dynamics model of damper-line system. The curve fitting of researchers is not as ideal as expected due to a large of parameters needed to determine in wind tunnel experiment. Now the more recognition and application is the wind power fitting function conducted by Rawlins C. [4].

\section{B. Self-damper Vibration Experiment of Line}

Self -damper power consumption of line needed to be provide in the energy balance method. IEEE [10] issued the corresponding experimental procedure, introduced two methods of self-damper experiment: free vibration method and forced vibration method. For the reason that self-damper of line in free vibration is small, procedures recommended power method and standing wave ratio method based on forced vibration method .Xiangzhi K. [11] come up with shear method to test the self-damper of line. Naiguan X. [12] summarized the damper formula for China's line based on a lot of experiments and the data fitting, and confirmed the formula by international verification. Zhixiong Y. put forward the improved formula of self-damper of transmission line and verified its rationality through the test.

\section{Indoor Test of Damper-Line System}

Energy balance method need measure the wind energy input of each part without considering many factors such as the location of damper, traveling wave, force transfer mechanism and coupling effect of damper-line system. Many scholars proposed dynamic research method to solve those flaw. Qiyu Zhen [13] conducted the indoor simulation to test the damper performance of UHV transmission line with consider the coupling effect of damper-line system and judged the cons and pros of anti-vibration scheme by comparing the maximum dynamic bending strain values of transmission line with in different anti- vibration scheme with the maximum strain limit in specification. Xiaoxiong $\mathrm{H}$, Lingfei J. [14] did the experimental study and on-line monitor for the Aeolian vibration of Xunjiang large span transmission line, and preliminary discuss the coupling property of damper and transmission line from two respects: the frequency and converted vibration mass aspects. Changju X. [15] perform indoor simulation test with optical fiber composite ground wire(OPGW) to study the anti-vibration effect in different parameter of the types of damper and its install location, direction. Jialin C. [16] studied anti-vibration problem of $1000 \mathrm{kV}$ UHV large span split conductor, analyzed the factors which influence the balanc vibration of UHV and the measure should be take, perfected the experimental condition and 
method, compared effect of two anti-vibration schemes which are whole stockbridge damper and the combination of the Bate damper line and stockbridge. Shengchun L. [17] designed the test of damper-line system which meet the characteristic of the dynamic method to explore the effect of the number, type, location of damper. Zhixiong Y. [18] verified the scientific of damper install location through the indoor experiment. Though experiment is the most close to the real situation of transmission line, experimental apparatus and experimental conditions are limited. With the increasing of span, experimental length is less than $34 \mathrm{~m}$ while the actual length is between $100 \mathrm{~m}$ and $100 \mathrm{~m}$. Furthermore, the experiment model is too simplified which cannot simulate the variability of regional environment [19].

\section{Dynamic Research of Aeolian Vibration}

The new trend of analyzing the dynamic property of Aeolian vibration is by studying the anti-vibration of damper -line system, which refers to building the kinetic equation of the damper-line system, the results of the equation are the dynamic response of the system. Some methods are used including the mode superposition method, wake oscillator model, finite difference method, and CFD simulation.

Claren, N. and Diana, G. [20] discussed the method of superposition of vibration mode to estimate the response of the transmission line which were subjected to excitation, but the simplified model only concerned about the hinge model, which restricts the range of its application. J. Vecchiarell [21] combined the finite difference method and the ideology of energy balance to calculate the single line vibration, after comparing the calculations and the test results, the conformity shows a wide application prospect. The mode superposition method as well as the finite difference method is to break up the system into two sub-system for study, one is the vortex-excited sub-system, other is hammer-line sub-system, the two sub-system are coupled by the input of the wind power. This method provides a simpler way to analyse the system with low calculation cost, but heavily depend on the wind tunnel test results. Oumar Barry [22]-[24] proposed a new damper-line model by Hamilton principle, the model showed the elastic-coupling of the damper-line system, meanwhile estimated the kinetic response between the line and thedamper, experiment results have proved the accuracy of the model. The model concerned on the relationship between the damper and line, but ignore the influence of the wind speed.

Wake oscillator model deals with the relationship of fluid area and the solid area directly. Bishop and Hsso [25] firstly proposed the wake oscillator model, regarding the influence of the Wake oscillator to structure as a nonlinear oscillator. Matteoluca, Emmanuel [26] summarized the wake oscillator model and the experiment result in recent 30 years, improved the wake oscillator model based on the Van De Pol equation. But due to the mode require significant amount of experimental data, it is not used widely. Williamson. $\mathrm{C} \mathrm{H}$, Govardhan. R. [27], Gabbai, R. D., Benaroya, H. [28] reviewed research on the Cylinder fluid-solid coupled vortex induced vibration in recent 20 years, thinking that CFD simulation is a new method to study on the hydrodynamics problems, but still cannot replace the wind tunnel experiment, so it's hard to apply the CFD method to simulate the damper-line Aeolian vibration.

\section{FATIGUE LIFE ANALYSIS OF AEOLIAN VIBRATION}

Transmission line installed with damper can notably reduce the response of Aeolian vibration. But due to the high frequency of Aeolian vibration, other issues may come up, in which the strand-broken and the abrasion problems are prominent. Different dynamic flexure stress lead to different fatigue life. Domestic researches about fatigue life are rare, design of the transmission line only obey the single limit principle and infinite life design method, which provide no quantitative analysis, as well as the fatigue lifetime estimation. Regulations about the range of the measurement are made in 1966 by IEEE[10], by gauge the dynamic flexure stress of the part of the line which is $89 \mathrm{~mm}$ away from clamps end, if $\varepsilon<150 \mu \varepsilon$, then ignore the fatigue damage, the limitation is loosened to $200 \mu \varepsilon$ in China. Jingpeng H. [29] concluded the fatigue properties of ACSR transmission line by simulation experiment under stable amplitude vibration and unstable amplitude vibration, the results prove the conservation of the Miner principle. Based on the Miner fatigue theory and the Wholer secure boundary curve proposed by the CIGRE 22nd committee, Jingchao W. [30] discussed the fatigue experimental method of wire and the lifetime estimation of the overhead transmission line under the composite strain-stress. Meng Z. [31] analyzed the transmission line fatigue life by Miner fatigue principle and the Wohler secure boundary curve Bin Z. [32] discussed the reasons causing the fatigue damage on the transmission line, but not giving the estimation of the lifetime and preventative measures. Li Q. [33] analyzed the vibration fatigue damage using the multi-function experimental system, concluded that fatigue damage and abrasion problems are the main issue of the transmission line, the part near the clamps end mainly damaged by stress fatigue, the vibration antinode part damaged by friction. Busheng $\mathrm{T}$ [34] improved the line fatigue accumulation model based on the Miner cumulative damage theory, designed the wireless net transmission line under Aeolian vibration monitoring system, the monitoring data results are consistent with real trail.

Domestic research on Aeolian vibration are limited by the following reasons: rare research results, lack of experimental data, and lack of experimental funding.

\section{Online Monitor Analyse of AEOLian Vibration}

Aeolian vibration are so complicated that the researches in laboratory can only simulate the wind field closely, but in the real situations, other circumstances far more complex will influence the results. DL/T741-2010 Operation rules for overhead transmission lines says, cable with long-span structure part should be gauged its vibration. Through the online monitor of Aeolian vibration, cable can be analysed according to the monitoring results, and given the proper solutions. Naiguan X. [35] viewed the technology of the long-span bundle conductor vibration experiment, including the measure technology, experiment procedure, calculation of 
the line strength, and the optimization of the anti-vibration methods. Xiaoxiong H. [15] confirmed safety of the technology according to the IEEE overhead vibration measurement on the Pingwer-Luohe $500 \mathrm{kV}$ transmission project.

Jinyu Y. [36] produced an online long-span cable measure system by a monitor and network, it also analyse the monitoring data, which play an irreplaceable part in the cable working, design improvement and the lifetime estimation. Meng Z. [31] designed an online observation system based on ZigBee technology, which will monitor the vibration of cable in the real time, and estimate the fatigue damage through field data. When monitoring the Yongxing long-span cable, Xiao S. [37] analyzed the Micro Meteorology and the Aeolian vibration data, pointed that the amplitude of the vibration will increase in low temperature, and propose that damper line and strock-bridge damper should be tightened to prevent the Grip relaxation. Hong K. [38] proposed and mounted a new type of damper to prevent the slip after installing the anti-vibration hammer. Haibo C. [39], Xiao S[40] pointed out that the online monitor focus on the record of vibration, frequency, wind speed, wind orientation, temperature, humidity, in the part where cable and clamps connect, further analysed on fatigue life will be approached through the record.

Researches about online monitoring in recent 20 years shows that the field test on the transmission line are rare, the application of the monitoring system is narrow.

\section{CONCLUSION}

This paper discuss the Aeolian vibration of the transmission line-damper system in the following three aspects: the experimental method of Aeolian vibration, the fatigue life estimation, and online monitoring system. Having probed into the recent research, several aspects worth further exploring:

1) Study more deeply on dynamic method based on the energy balance method, probe into the theoretical calculation formulas, the properties of the damper power and impedance, the damper-line system dynamic analysis method.

2) Based on the transmission line experiments, build up the damper- line system numerical model on vibration, discuss the mechanism of Aeolian vibration.

3) Build up the database of the cable response under Aeolian vibration, acquire abundant test data to support theoretical analysis.

4) Structure the model of fatigue damage of the transmission line under Aeolian vibration, study the fatigue loading spectrum to estimate the fatigue lifetime, analyse the vibration using fatigue method.

5) Online vibration monitoring system on significant transmission line is gradually moving towards maturity and practicability. Utilizing the real time monitoring data to estimate the safety of the transmission line and the anti-vibration design is playing an irreplaceable role.

\section{ACKNOWLEDGMENT}

The financial support from Guangdong Electric Power
Company Research Institute is gratefully acknowledged.

\section{REFERENCES}

[1] H. J. Song, L. Lan, W. A. Ping, and W. D. Jun, "The main technical principles of feasibility studies on UHVDC transmission projects," Electric Power, vol. 40, no. 8, pp. 36-39, 2007

[2] T. X. Shao, Power Calculation of Overhead Transmission Line, Beijing: China Electric Power Press, 2003.

[3] S. M. Meng, Design of Transmission Line, Beijing: China Electric Power Press, 2007.

[4] C. H. Williamson and R. Govardhan, "Vortex-induced vibrations, annual review of fluid," Mechanics. vol. 36, no. 1, pp. 413-555, 2004

[5] C. Rawlins, Model of Power Imparted to a Vibrating Conductor by Turbulent Wind, Alcoa Conductor Products Company, 1983.

[6] R. Macdonald, C. J. Pon, D. G. Havard et al., Tech. Report, Canadian Electrical Association Aeolian Vibration Excitation of Bundle Conductors, 1989.

[7] D. Brika and A. Laneville, "A laboratory investigation of the Aeolian imparted to a conductor using a flexible circular cylinder," IEEE Transactions on Power Deli, vol. 2, no. 11, pp. 1145-1152, 1996.

[8] M. Kraus and P. Hagedorn, "Aeolian vibrations: Wind energy input evaluated from measurements on an energized transmission line," IEEE Transactions on Power Delivery, vol. 3, no. 6, pp.1264-1270, 1991.

[9] D. Kong, UHV Transmission Line Aeolian Vibration Research Based on Dynamic Method, Wuhan: Huazhong University of Science and Technology, 2009.

[10] IEEE Guide on Conductor Self-Damping, IEEE Committee, 1978.

[11] X. G. Kong, "Ways to measure self-dampering of aerial conductor," Journal of Civil Aviation Institute of China, vol. 3, no.10, pp. 34-41, 1992.

[12] N. G. Xu and J. C. Wang, "Conductor self-damping measurement and a practical calculating method," Electric Power, vol. 2, no. 28 pp.17-20, 1995.

[13] Q. Y. Zhen, Aeolian Vibration of Transmission Lines, Beijing: China hydraulic Press, 1987.

[14] X. X. He and L. F. Ji, "Field measurement of Aeolian vibration of Pingwer-Luohe $500 \mathrm{kV}$ transmission line," Journal of Hefei University of Technology, vol. 4, no. 24, pp.555-558, 2001.

[15] C. J. Xie, Z. J. Zhang, and M. Li, "Experiments and field measurements of damper designed for breeze vibration of a transmission line across Xunjiang river," World Earthquake Engineering, vol. 2, no. 19, pp. 82-86, 2003.

[16] J. L. Chen and J. K. Li, "Vibration preventing of OPGW," Telecommunications for Electric Payer System, vol. 9, no. 27, pp. 6-9, 2006.

[17] S. C. Liu, K. J. Zhu, H. Wang et al., "Research on Anti-vibration for 1000 kV UHV transmission lines," High Voltage Engineering, vol. 4, no. 34, pp. 638-642, 2008.

[18] Z. X. Ye, Studies of Aeolian Vibration and Subspan Oscillation Control, Wuhan: Huazhong University of Science and Technology, 2009.

[19] R. M. Brunair, G. E. Ramey, and R. R. Duncan, "Experimental evaluation of S-N curves and validity of Miner's cumulative damage hypothesis for an ACSR conductor," IEEE Transactions on Power Delivery, vol. 3, no. 3, pp. 1131-1140, 1988.

[20] R. Claren and G. Diana, "Mathematical analysis of transmission line vibration," IEEE Transactions on Power Apparatus and Systems, vol. 12, no. 88, pp. 1741-1771, 1969.

[21] J. Vecchiarelli, I. G. Currie, and D. G. Havard, "Computational analysis of Aeolian conductor vibration with a stockbridge-type damper," Journal of Fluids and Structure, vol. 4, no. 14, pp. 489-509, 2000.

[22] O. Barry, D. C. D. Oguamanam, and D. C. Lin, "Aolian vibration of a single conductor with a Stockbridge damper," Journal of Mechanical Engineering, vol. 227, no. 5,pp. 935-945, May, 2012.

[23] O. Barry, J. W. Zu, and D. C. D. Oguamanam, "Forced vibration of overhead transmission line, analytical and experimental investigation," ASME J. Vib.Control, vol. 136, no. 4, p. 041012, May, 2014.

[24] O. Barry, J. W. Zu, and D. C. D. Oguamanam, "Nonlinear dynamics of stockbridge dampers," Journal of Dynamic Systems, Measurement, and Control, vol.137, pp. 0610171-0610177.

[25] R. Bishop and A. Hassan, "The lift and forces on a circular cylinder in a flowing fluid," Royal Society of London Proceedings, vo1. 638, no. 277, pp. 32-50, 1964. 
[26] F. Matteoluca and D. L. Emmanuel, "Coupling of structure and wake oscillators in vortex-induced vibrations," Journal of Fluids and Structures , vol. 1, no.1, pp. 116-133, 2004.

[27] C. H. Williamson and R. Govardhan, "Vortex-induced vibrations," Annual Review of Fluid Mechanics, vol. 36, pp. 413-455, 2004

[28] R. D. Gabbai and H. Benaroya, "An overview of modeling and experiments of vortex-induced vibration of circular cylinders," Journal of Sound and Vibration, vol. 3, no. 282, pp. 575-616, 2005.

[29] J. P. Hou, X. H. Xu, and Z. T. Sun, "Advances in the research on wind oscillation of vibration damper-transmission system," Journal of Hefei University of Techology, vol. 5, no. 34, pp. 743-747, 2011.

[30] J. C. Wang, G. N. Xu, and K. J. Zhu, "Conductor fatigue test method of composite alternating stress," Electric Power Construction, vol. 3, pp. $18-20,2001$.

[31] M. Zhang, Online Mornitor System Reaearch and Application of Aeolian Vibration in Transmission Line, Taiyuan university of technology, 2012

[32] B. Zhu, "Fatigue failure of transmission line and its prevention," Rural Electrification, vol. 5, pp.12-13, 2005

[33] L. Qin and Y. Xu, "Study on fatigue test of steel reinforced Aluminium conductors for transmission lines," Water Resources and Power, vol. 8, no. 31, pp. 181-183, 2013.

[34] B. S. Tong, Aeolian Vibration Monitor and Fatigue Damage Research of Transmission Line, Taiyuan university of technology, 2013

[35] N. G. Xu and J. C. Wang, "Experimental research on anti-vibration of 500 kV long-span transmission tower," Electric Power Construction, vol. 11, no. 15, pp. 2-5, 1994.

[36] Y. J. Yang, "Aeolian vibration monitor of 500kV transmission line," Anhui Electric Power, 200

[37] X. Su, "Application of on-line monitoring technologies for UHV AC transmission lines," Electronic Technology and Software Engineering, vol. 1, pp. 131-132, 2015.

[38] H. Kang, W. Y. Yu, and H. Yu, "Research on shifting rate reduction of stockbridge damper of overhead transmission lines," Zhejiang Electric Power, vol. 8, pp. 62-64, 2015.

[39] H. B. Chen and C. Wang, "Application of on-line monitoring technologies for UHV AC transmission lines," Power System Technology, vol. 10, no. 33, pp. 55-58, 2009.

[40] X. Su, "Application of on-line monitoring technologies for UHV AC transmission lines," Electronic Technology, pp. 131-132, 2015.

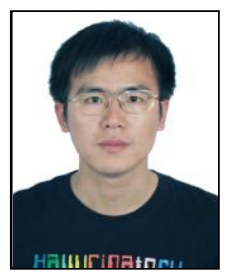

Wenping Xie was born in Jiangxi province, China, in 1986. He received the master degree in engineering mechanics from Huazhong University of Science and Technology, Wuhan, China. in 2012

Currently, he work in Electric Power Research Institute of Guangdong Power Grid Co., Ltd, Guangzhou, 510080, China. His currently research interests include structure fatigue research, structural optimization of power equipment and power system overvoltage analysis.

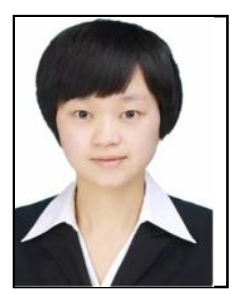

Tian Peng was born in Jiangxi province, China, in 1992. She is a second-grade postgraduate in civil engineering in Tongji University, Shanghai, China.

Her currently research interests include structure fatigue research, Aeolian vibration research of transmission lines, wind resistance of transmission lines.

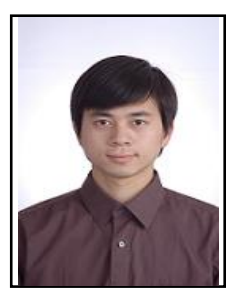

Kai Xiao was born in Hunan province, China, in 1986. He received the master degree in materials science and engineering from Zhejiang University, Hangzhou, 310000 , China in 2011.

Currently, he work in Electric Power Research Institute of Guangdong Power Grid Co., Ltd, Guangzhou, 510080, China. His currently research interests include structural optimization of power equipment and power system overvoltage analysis and monitoring technology of transmission lines.

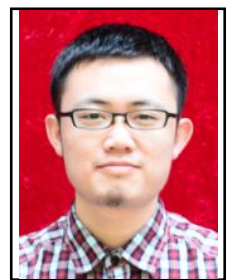

Jian Zhang was born in Inner Mongolia, China, in 1992. He is a first-grade doctoral students in civil engineering in Tongji University, Shanghai, China.

His currently research interests include wind resistance of transmission lines, transmission tower strengthening, transmission tower-line system analysis. 